\title{
Study of Clinico-Epidemiological Correlation and Clinical Pattern of Cutaneous Drug Reaction in Tertiary Level Hospital in Western Rajasthan
}

\author{
Virendra Kumar*, Vinod Jain, Dilip Kacchawaha, Pankaj Rao and C.P. Chouhan \\ Department of Skin \& V.D, Dr. S.N. Medical College, Jodhpur, Rajasthan, India \\ *Corresponding author
}

\section{A B S T R A C T}

\begin{tabular}{|l|}
\hline Ke y w o r d s \\
Adverse cutaneous \\
drug reaction, \\
Maculopapular \\
rash, Toxic \\
epidermal \\
necrolysis, LDR, \\
DRESS \\
Article Info \\
\hline $\begin{array}{l}\text { Accepted: } \\
\text { 20 May } 2020 \\
\text { Available Online: } \\
\text { 10 June 2020 }\end{array}$ \\
\hline
\end{tabular}

Adverse drug reactions are described as "A response to a medicine in a human which is noxious and unintended and which occur at any dosage and can also result from an overdose, misuse or abuse of a medicine. Adverse cutaneous drug reactions (ACDRs) are caused by a wide variety of drugs. Aim of the study is to determine the clinical patterns of drug eruptions and the common drugs implicated and determine the frequency of incriminating drugs for different patterns of CADRs. The time duration of our study was about 6 months from January 2018 to December 2018 and 500 patients attending the dermatology OPD and IPD of MDM hospital were included in this study. All patients were examined clinically and history and informed consent were taken. All routine and any special investigations if needed were done and pro forma filled. The most common drug rectoins pattern was fixed drug eruptions in 220 (44\%) patients followed by maculopapular rash in $115(23 \%)$ patients, urticaria in $45(9 \%)$, urticarial vasculitis in $15(3 \%)$ and SJS in $30(6 \%)$ patients. TEN and EM like in $25(5 \%)$, LDR, Erythroderma, PR like, acneiform eruption and DRESS in $5(1 \%)$ patients. The clinical patterns and causative drugs of cutaneous adverse drug reactions (CADR) are highly variable among the population. Thus a thorough knowledge of these drug eruptions and the causative drugs will help the clinician in minimizing the ACDRs and also in its timely management.

\section{Introduction}

According to WHO, Adverse drug Reaction (ADR) - "A response to a drug which is noxious and unintended and which occur at doses normally used in man for the prophylaxis, diagnosis or therapy of disease or for the modifications of physiological function. Cutaneous adverse drug reactions are responsible for the majority of ADRs in hospitalized patients ${ }^{1}$. Any untoward medical occurrence associated with the use of a drug in human, whether or not considered drug related. Any unfavorable and unintended sign (e.g. An abnormal laboratory finding), symptom or disease temporally associated with use of drugs, without any judgment about causality ${ }^{2}$. Cutaneous ADRs account for approximately $14 \%$ of ADRs in hospital patient and $3 \%$ of all disabling injuries during hospitalization ${ }^{3}$.

Despite development in once of ADRs in relay decader data on prevalence and related 
factors of hypersensitivity reaction, in population to drugs in the outpatient population remain sponse. Most reports on drug reaction focused on in-patient cases, whereas drug hypersensitivity reaction has been reported to affect more than $7 \%$ of general population ${ }^{4}$. The incidence of adverse cutaneous drug reaction varies from $2-5 \%$ of hospitalized patient and approximately 10 in 1000 hospital patients suffer from life threatening cutaneous drug reactions. The most frequently observed are cutaneous reactions and that range from generally trivial manifestation, such as pigmentation (hypo and hyperpigmentation) to severe life threatening reaction such as Toxic Epidermal Necrolysis (TEN), Steven Johnson Syndrome, Exfoliative Dermatitis (ED), Drug Hypersensitivity Syndrome (DHS).Their importance is significant in terms of cost and health service resources ${ }^{6}$. Majority of cutaneous ADRs are minor reactions which are self-limiting. Sometimes, severe and potentially life threatening situation like Steven Johnson Syndrome (SJS) and Toxic Epidermal Necrolysis (TEN) can occur which constitute from 2.6 to $7 \%$ of all drug reaction ${ }^{7}$.

Stevens Johnson Syndrome (SJS) and Toxic Epidermal Necrolysis (TEN) are the most severe form of cutaneous adverse drug reaction. The most common culprits are nonsteroidal anti-inflammatory drugs, anticonvulsant, allopurinol, sulfonamides, antifungal and antibiotics ${ }^{8}$.

\section{Materials and Methods}

The study was conducted at Department of Dermatology, Venereology and Leprosy, Dr. S. N. Medical College, Mathura Das Mathur Hospital, Jodhpur $\left(3^{\text {rd }}\right.$ level hospital of Western Rajasthan) respectively, for around 12 months 500 patients attending the dermatology OPD and IPD of MDM hospital were included in this study. All patients were examined clinically and history and informed consent were taken. All routine and any special investigations if needed were done and proforma filled.

\section{Adverse drug reaction assessment}

An observational cross sectional study was undertaken in the Dermatology out Patients Department (OPD) of Mathura Das Mathur Hospital, Jodhpur, for period of $12^{\text {th }}$ months. All the patients visiting the Dermatology out Patients (OPD) Department between January2018 to December2018 with any suspected cutaneous adverse drug reaction was included in the study.

\section{Causality assessment}

Casualty assessments of the patients were done by two scales ${ }^{10}$ :

\section{Naranjo's scale}

Severity scale -Modified Hartwig and Siegel scale of ADRs

\section{Results and Discussion}

The study was conducted in the Department of Dermatology, Dr. S. N. Medical College and Department of Dermatology, Mathura Das Mathur hospital, Jodhpur, for a period of around 12 months. This study was prospective, cross sectional and observational. Each patient was assessed frequently during the study course. A total of 500 patients with suspected cutaneous adverse drug reactions were recruited during the study period from January 2018 to December 2018. The majority of patients in whom cutaneous ADRs were observed belonged to age group $16-30$ years $(45 \%)$ followed by $31-45$ years $(30 \%),>45$ years $(20 \%)$ and $0-15$ years $(5 \%)$ respectively. Out of a total of 500 patients 310 
$(62 \%)$ were males and $190(38 \%)$ were females (Figure 1).

Most frequently reported cutaneous drug reactions were caused by NSAIDS in 175 (35\%) patients, followed by antibiotics in 135 (27\%) cases, antifungal in $40(12 \%)$ cases, antihypertensive drugs in $25(5 \%)$ cases and antitubercular drugs in $10(2 \%)$ cases. The most common drug groups implicated and the common cutaneous ADRs are shown in (figure 2). In our study a wide type of the cutaneous manifestation ranging from FD to dress occur. The most common drug reaction pattern was fixed drug eruptions in $220(44 \%)$ patients followed by a maculopapular rash in 115 (23\%) patients, urticaria in 45 (9\%), urticarial vasculitis in $15(3 \%)$ and SJS in 30 (6\%) patients. TEN and EM like in $25(5 \%)$, LDR, Erythroderma, PR like, acneiform eruption and DRESS in $5(1 \%)$ patients. The type of cutaneous ADR was shown in (Figure $3)$. In the present study most commonly reported, the spectrum of the cutaneous drug reaction was caused by NSAIDS in total 175 patients followed by antibiotics in 135 cases, antiepileptic in 60 cases, antihypertensive drug in 25 cases and antitubercular drug in 10 cases. The most common type of drug reaction pattern was fixed drug eruption in 220 patients followed by a maculopapular rash in 115 patients and urticaria in 45 patients. The spectrum of the pattern of CADRS was shown in (figure 4). The most frequently reported cutaneous drug reaction with implicated drugs was diclofenac (NSAIDS) (Figure 8) in 108 patients followed by ofloxacin (Figure 9) (Antimicrobial) in 76 patients, fluconazole antifungal in 27 patients, phenytoin (Antiepileptic) (Figure 10) in 26 patients, Isoniozide (ATT) in 10 patients, Nevirapine (ART) in 10 patients and chloroquine (antimalarial) in 5 patients. The spectrum of the ADRs with implicated drugs was shown in (figure 5).

Table.1 List of drugs commonly causing Cutaneous ADRs ${ }^{9}$

\begin{tabular}{|c|c|}
\hline GROUP & DRUGS \\
\hline Antibacterials & $\begin{array}{c}\text { Sulfonamides, Penicillins, Cephalosporins, } \\
\text { Quinolones, Vancomycin }\end{array}$ \\
\hline Anticonvulsants & $\begin{array}{c}\text { Phenytoin, Carbamazepine, Phenobarbitone, } \\
\text { Valproate, Lamotrigine }\end{array}$ \\
\hline NSAIDs & $\begin{array}{c}\text { Phenylbutazone, Piroxicam, Aspirin, Diclofenac } \\
\text { ATT drugs }\end{array}$ \\
\hline ART drugs & Nevirapine, Abacavir, Protease Inhibitor \\
\hline Anti-malarial drug & Chloroquine, Quinine \\
\hline Antifungal & Fluconazole, Itraconazole, Griseofulvin \\
\hline Anti-gout drug & Allopurinol \\
\hline $\begin{array}{c}\text { Immunosuppressor and } \\
\text { modulator }\end{array}$ & Methotrexate, Azathioprin, Cyclosporine \\
\hline Antihypertensive & Beta blocker, CCB, Diuretics \\
\hline Anticancer & Hydroxylene, Imatinib, Vinblastin, Vincristine \\
\hline Biological agents & TNF inhibitor, Rituximab \\
\hline Topical agents & Antibacterial, Antiviral, Retinoids, steroids \\
\hline Others & \\
\hline
\end{tabular}


Figure.1 Age wise distribution of cases

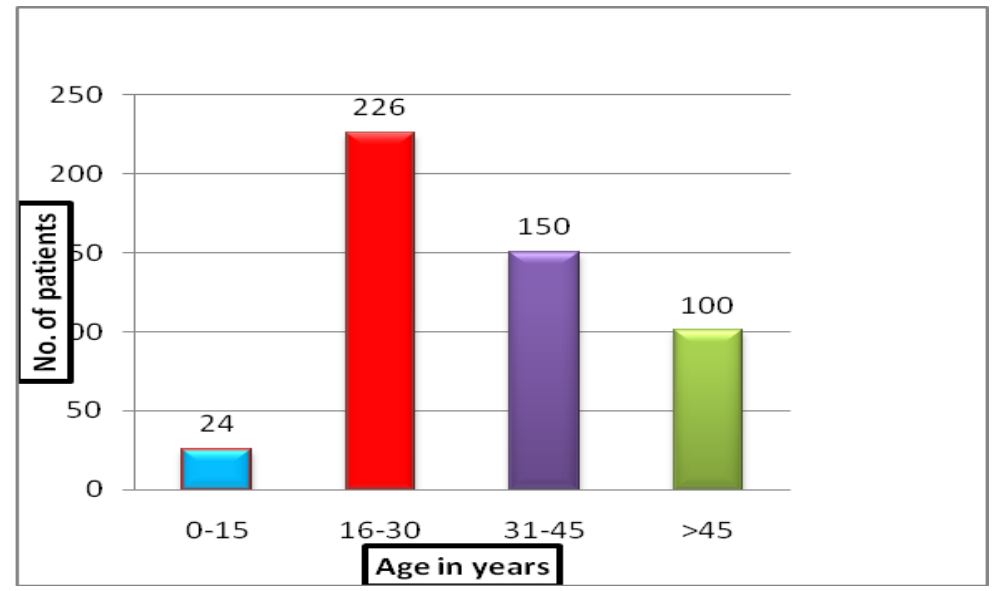

Figure.2 Sex wise distribution of cases

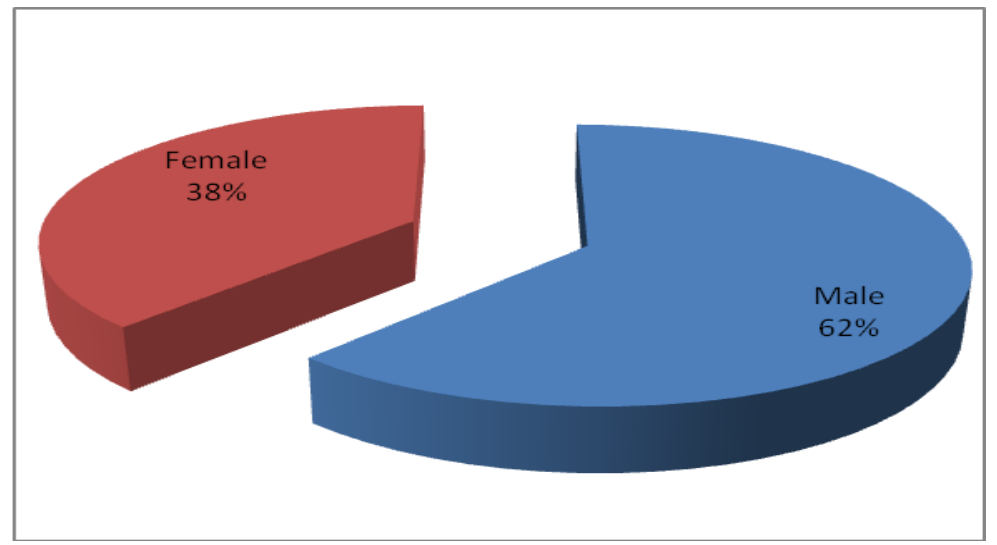

Figure.3 Drugs groups causing cutaneous ARDs

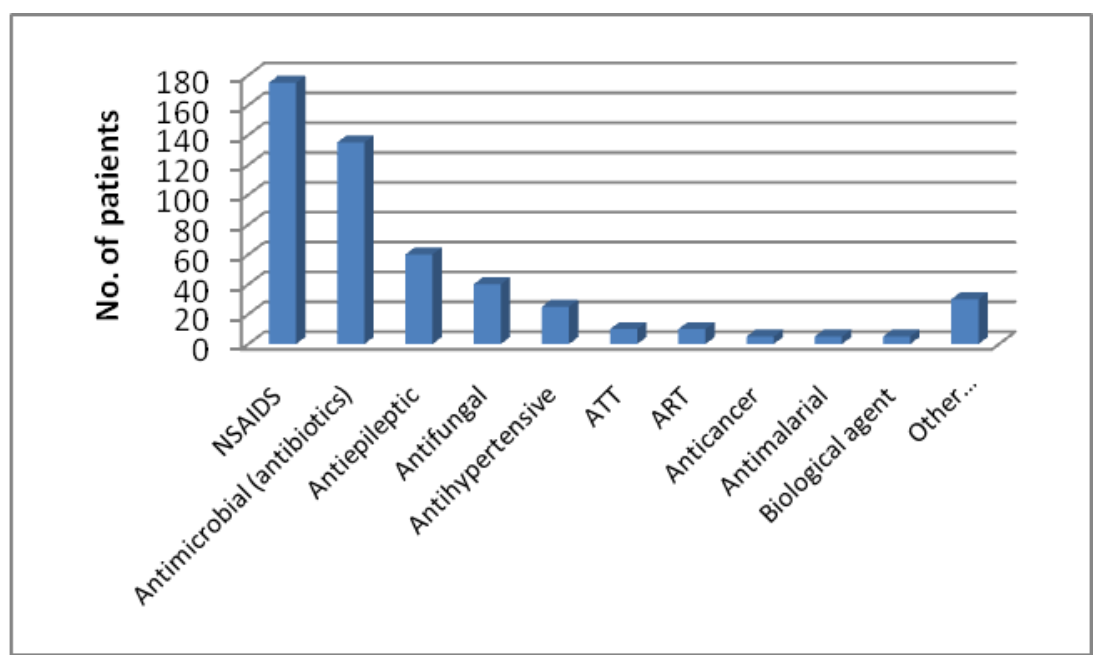

NSAIDs: Nonsteroidal Anti-Inflammatory Drugs, ART: Antiretroviral Therapy, ATT: Antitubercular Therapy 
Figure.4 Types of cutaneous ADRs

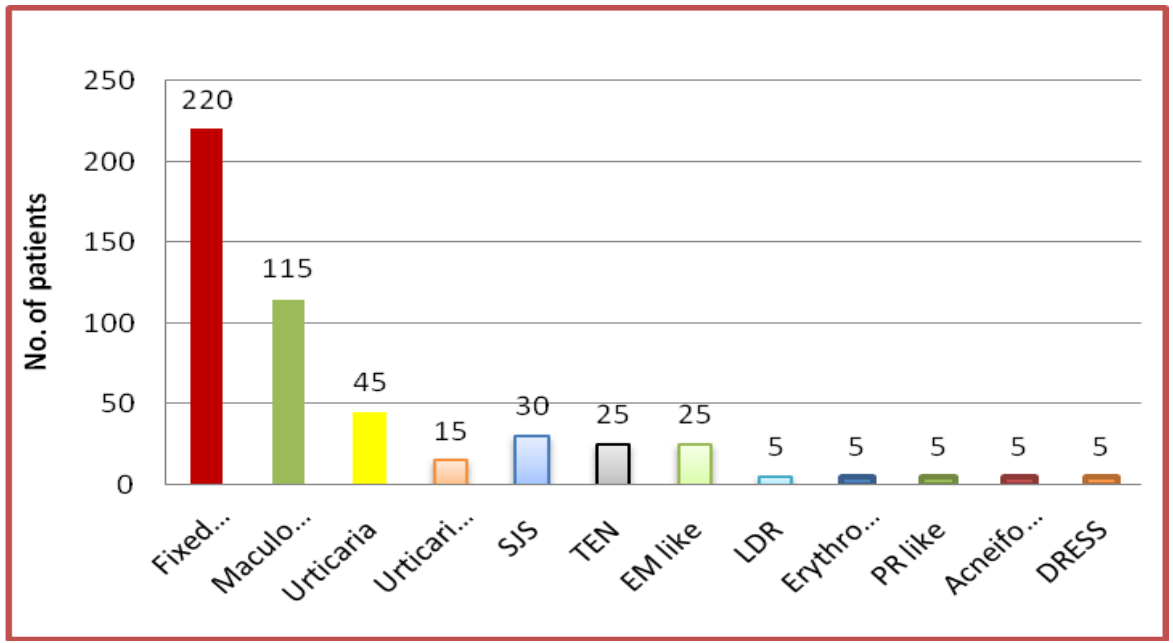

Figure.5 Spectrum of cutaneous ADRs

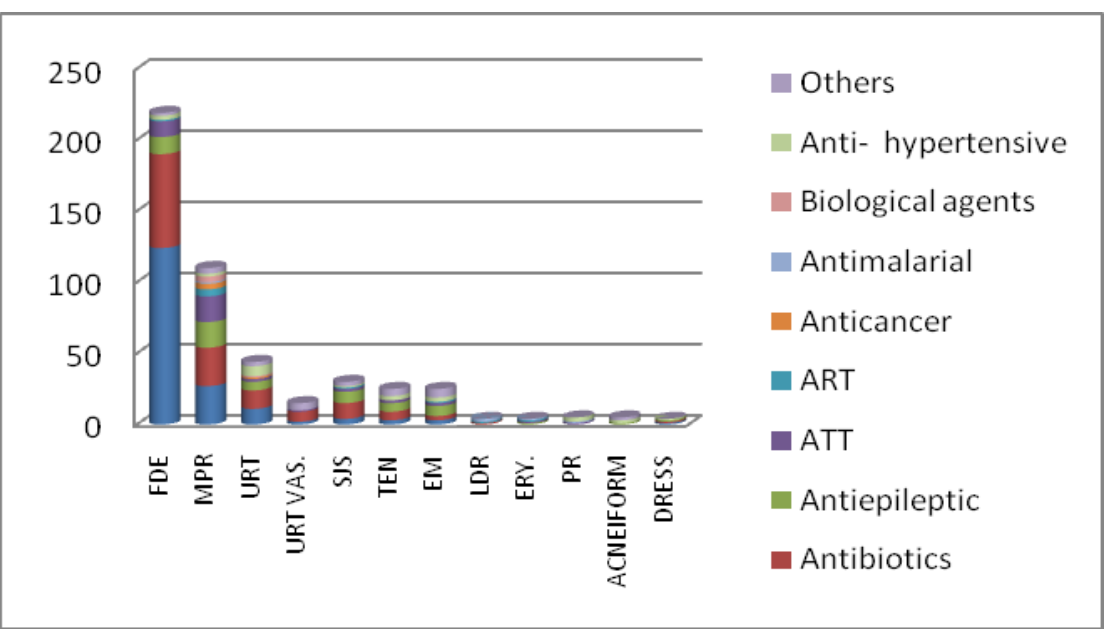

Figure.6 Naranjo's causality scale

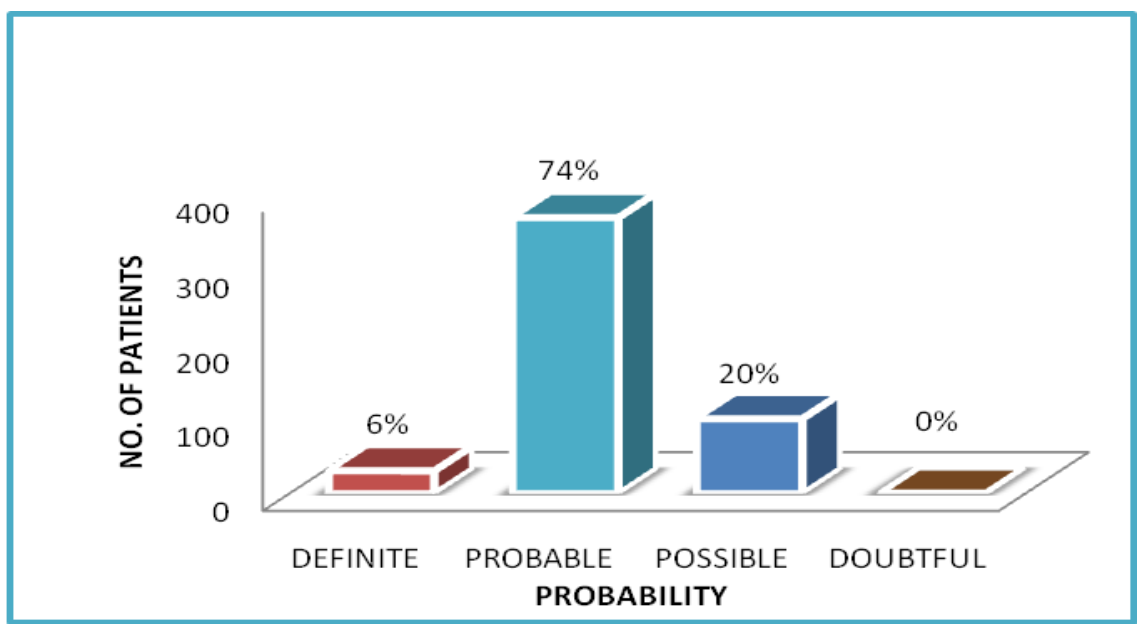


Figure.7 Number of ADRs

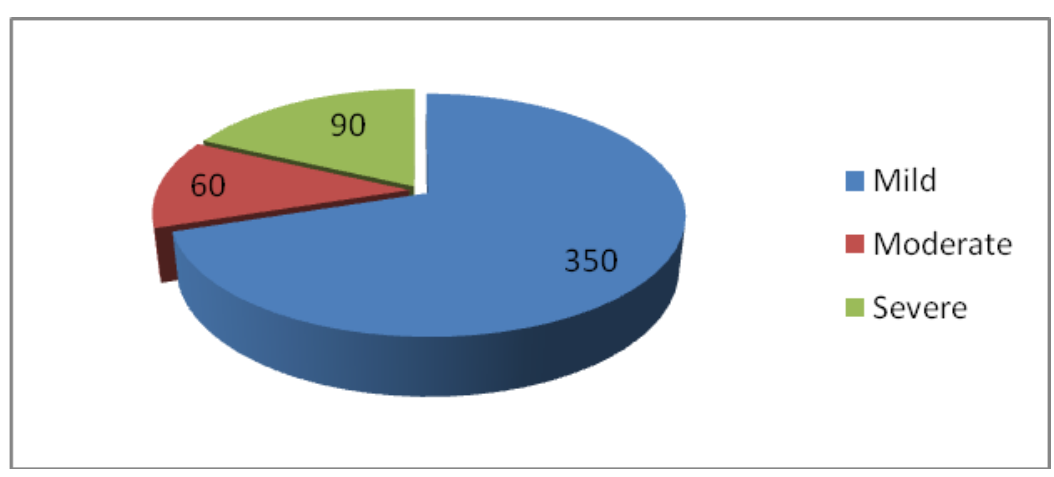

Figure.8 FDE due to diclofenac

Figure.9 Bullous FDE due to ofloxacin
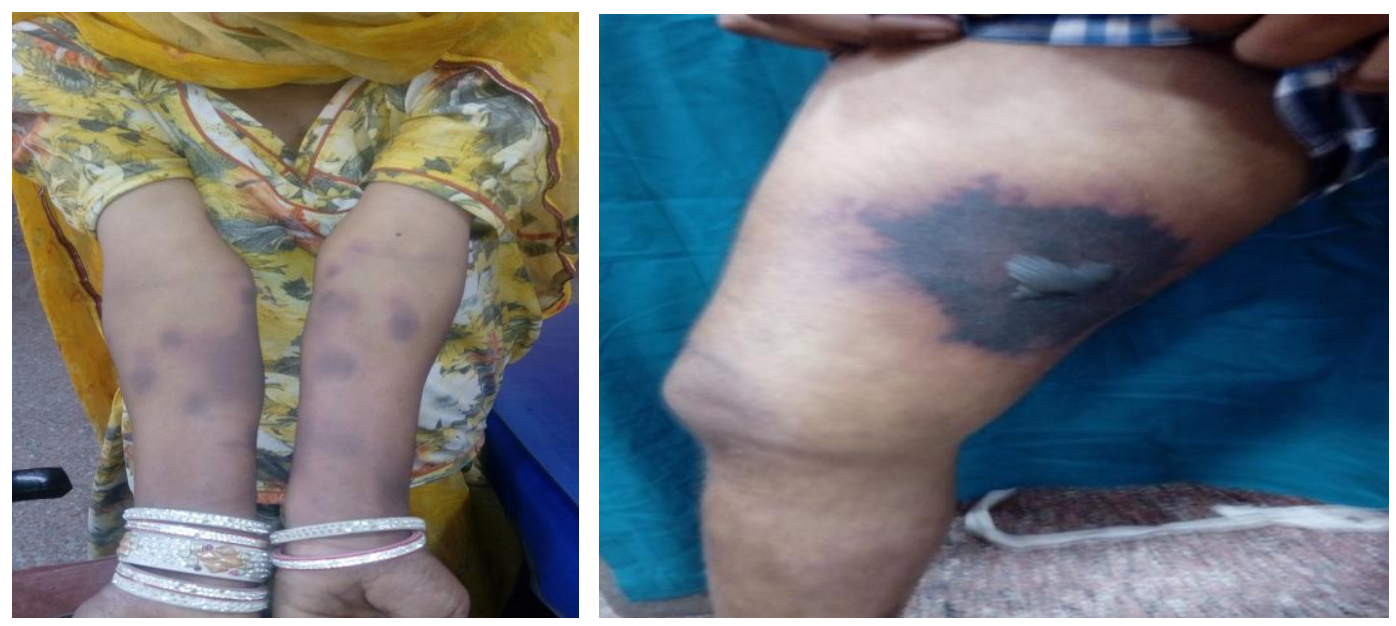

Figure.10 TEN due to valproate (Antiepileptic group)

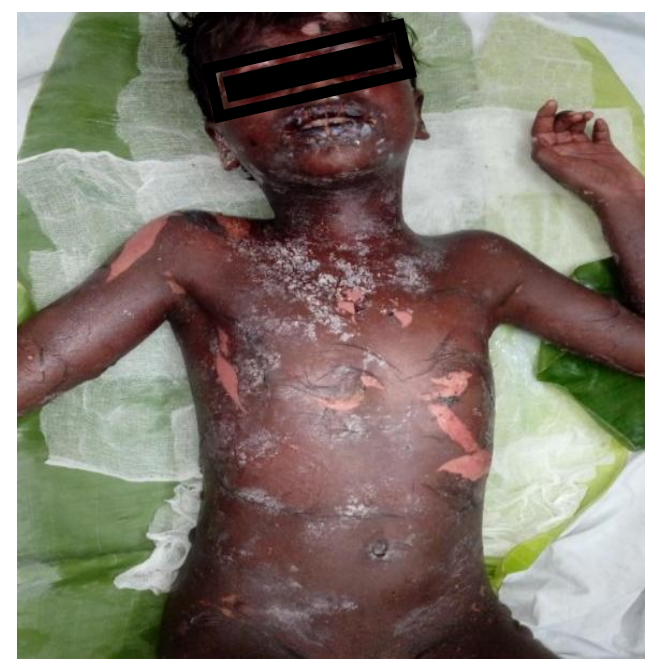




\section{Causality assessment scale}

The present study assessed the causality of the patients by Naranjo's causality scale. In study definite probability in 30 patients, probable in 370 patients, possible in 100 patients and doubtful probability in zero patients. The maximum probability was probably in $74 \%$ patients and minimum probability was doubtful in $0 \%$ patients. The Naranjo's causality scale of the assessment was shown in (figure no. 6).

\section{Severity assessment scale}

In this study assess the causality of the patients by Modified Hartwig and Siegel scaling. In the data analyzed mild level in 350 patients, severe in 90 patients and moderate in 60 patients. The maximum level was mild in $70 \%$ patients and minimum in moderate in $12 \%$ patients. The modified Hartwig \& Siegel scaling was shown in (Figure. 7).

Adverse cutaneous drug reactions are common and some can be dangerous with $0.2+0.30 \%$ of all CADR required hospitalization. Adverse cutaneous reactions are distressing for both patient and dermatologist. Mortality can occur in severe reaction, but even without this quality of life could be significantly diminished due to prolong hospitalization and increase morbidity. Probability of developing an adverse drug reaction to the new drug is increasing as more and more new drugs are available in the market. There are very few studies on adverse drug reaction in dermatology in an outpatient population in our country. Hence, the present study was conducted to observe the clinical pattern of drug induced cutaneous reaction in dermatology OPD.

\section{Demographic profile}

CADR were observed for a slightly more in males $62 \%$ as compared to female $38 \%$. Out of total 500 patients our results were similar to two studies done by Sharma et al., 2001 in Chandigarh where they also have reported a male predominance but these findings differ from a study by Saha et al., 2012. The mean age of our study population was 36.27 years and $45.2 \%$ of patient belonged to the age group of 16-30 Years which is similar to a study done by Saha et al., 2012 in a tertiary care hospital in Kolkata.

\section{Pattern of adverse drug reaction}

The clinical pattern of cutaneous ADRS with suspected drug was observed. The CADR manifested with varied and diverse morphological pattern ranging from maculopapular rash to severe reaction like SJS and TEN. FDE was the most common manifestation among cutaneous ADRs accounting for $44 \%$ patients, followed by a maculopapular rash in $23 \%$ patients, urticaria in $9 \%$, urticarial vasculitis in $3 \%$, SJS in $6 \%$, TEN and EM like 5\%, LDR, Erythroderma, PR like, Acneiform eruption and DRESS in $1 \%$ of the patients.

A high incidence of FDE and MPR has also been reported from various other Indian studies conducted by Saha et al., 2012, Pudukodan and Thappa, 2004, However, the incidence of FDE and MPR was found to be lower in Western studies. In contrast to our findings where fixed drug eruption was found to be most common cutaneous ADR a study conducted by Sharma et al., 2001 and Pudukadan and Thappa, 2004. NSAIDS were the most common drug group which cutaneous ADR (35\%) followed by antibiotics (27\%) and antiepileptic (12\%) which was consistent with the finding of other studies done in India and China. In our study NSAIDS were mainly implicated in mild to moderate cutaneous ADRs like FDE, MPR, urticaria and urticaria vasulitis. NSAIDS mainly responsible for these ADRs were 
diclofenac, ibuprofen and Mefenamic acid caused a wide spectrum of cutaneous ADRs. Phenytoin and carbamazepine were responsible for most of the severe cutaneous ADRs like SJS and TEN.

Our study showed that reaction time for various cutaneous ADRs ranged from a few hours to 180 days with mean reaction time 5.40 days. The reaction time is the time interval between drug intake and first appearance of cutaneous lesions. The reaction time for maculopapular rash, fixed drug eruptions, Acneiform eruptions and urticaria varied 1 to 10 days and reaction time for SJS and TEN ranged from 8 to 70 days. This profile of reaction time is similar to the study by Sushma et al., 2005 but slightly different from the study by a Sharma et al., 2001. Taking into account the different drugs and their respective reaction times, it appears that antibiotics and NSAIDs tend to have short reaction time whereas antiepileptics and allopurinol have a longer latency period. This shows that not only doctor needs to inquire about recent medications, but also it is important that the doctor should be vigilant about CADR even to drugs patients are taking from the long period (especially for phenytoin, carbamezipine and allopurinol). The reaction time can also be helpful in suggesting the offending drug in cases of polypharmacy which in turn will prevent unnecessary withdrawal of harmless medicines.

In our study, dechallange was done in most of the cases. In our study rechallange was not attempted in any of the patients because of the possible associated risks of more severe reaction after rechallange with the suspected drugs. The patterns of the cutaneous adverse drug reactions and the drugs implicated variables according to the pattern of the drug intake, the associated illness and the susceptibility of the patients. A good knowledge of the adverse drug reactions, a careful history taking and a watchful approach while prescribing of new drugs can prevent many of the adverse drug reactions. As newer drugs are entering the market promptly special attention must be given to monitor and report the cutaneous adverse drug reactions.

Hence, concluded that the study was varying focused on clinical patterns of drug induced cutaneous reaction in the dermatology outpatient department of MDM hospital. A wide range spectrum of CADRS was observed ranging from essential and maculopapular rash to severe reaction like SJS and TEN. The commonest type of cutaneous ADR observes was FDE 44\%, followed by maculopapular rash $23 \%$, urticarial $9 \%$ and urticarial vasculitis $3 \%$ etc. NSAIDS $35 \%$ were most common drug which were implicated for cutaneous ADRS in our study, followed by antibiotic $27 \%$, antiepileptic $12 \%$ and antifungal $8 \%$. Most of drug reaction cause by drug Diclofenac and Ofloxacin were responsible for most of severe cutaneous ADR like FDE, MPR and urticarial. The reaction time for various ADRS ranged from a few hours to 180 days. In our study, dechallange was done in most of the cases and rechallange was not attempted in any of the patients because of ethical reasons. So, good knowledge of adverse drug reactions, a careful history taking and a watchful approach while prescribing of a new drug can prevent many adverse drug reactions.

Adverse drug reaction constitutes a major clinical problem in terms of increase in morbidity and mortality as well as an increase in cost and health care. Adverse drug reaction may affect any organ and skin is the most common site of presentation. Majority of cutaneous ADRS is minor reaction and selflimiting, but sometime severe and potentially 
life threatening reaction like SJS and TEN can also occur. Most of the reports on cutaneous drug reaction focused on inpatient cases, whereas it has been reported that skin reactions affect a large number of patients in the general population.

The cutaneous adverse drug reaction manifested with varied and diverse morphological pattern ranging from maculopapular rash to severe reaction like SJS and TEN. FDE was the most common manifestation among ADRS followed by maculopapular rash, urticaria and urticarial vasculitis, SJS and TEN. NSAIDS were the most common drug group which caused cutaneous ADRs (35\%), followed by antibiotics (27\%) and (12\%), respectively. The reaction time for various cutaneous ADR ranged from a few hours to 180 days. The reaction time for maculopapular rash, fixed drug eruptions, acneiform eruptions and urticaria varied 1 to 10 days and reaction time for SJS and TEN ranged from 8 to 70 days.

\section{References}

1. WHO. International drug monitoring: The role of national centres. Tech rep ser WHO 1972, no. 498. Available on internet at [http://www.whoumc.org/graphics/24756.pdf]

2. Kramer MS, Leventhal JM, Hutchinson TA, Feinstein AR. An algorithm for the operational assessment of adverse drug reactions. I. Background description and instruction for use. J Am Med Assoc 1979; 242: 623-32.

3. Valeyrie-Allanore L, Sasselas B, Roujeau JC. Drug-induced skin, nail and hair disorder. Drug saf 2007; 30:1011-30.

4. Demoly P, Gomes R. Drug hypersensitivities: definition, epidemiology and risk factor. Eur Annallergy clin Immunol 2005; 37:202-206.

5. Fiszenson AF, Auzerie V, Mahe E, Farinotti R, Durand SC, Cricks B et al., A 6 month prospective survey of cutaneous drug reaction in a hospital setting. Brj dermatol 2003; 149: 118-22.

6. Roujeau JC, Stem RS. Severe adverse cutaneous reactions to drugs. N Engl J Med 1994; 331: 1272-85.

7. Naldi L, Conforti A, Venegoni M, Troncon MG, Caputi A, Ghiotto E, et al., Cutaneous reactions to drugs. An analysis of spontaneous reports in four Italian Regions. Br J Clin pharmacol 1999; 48:839-46.

8. Roujeau JC, Kelly JP, Naldi L, Rzany B, Stem RS, Anderson T, et al., Medication use and the risk of Stevens-Johnson Syndrome or Toxic Epidermal Necrolysis. N engle J Med 1995:333:1600-07.

9. Chan HL, Stern RS, Arndt KA, et al., The incidence of erythema multiforme, Stevens Johnson Syndrome, and Toxic Epidermal Necrolysis. A population based study with particular reference to reaction caused by drug among outpatients. Arch dermatol 1990; 126: 43-47.

10. Naranjo CA, et al., pharmacol. Ther. (1981). $30 ;(2): 239-45$

11. WHO. International drug monitoring: The role of national centres. Tech rep ser WHO 1972, no. 498. Available on internet at [http://www.whoumc.org/graphics/24756.pdf]

12. Sharma VK, Sethuraman G, Kumar B. Cutaneous adverse drug reactions: Clinical pattern and causative agents - a 6 year series from Chandigarh, India. J Postgrad Med 2001; 47:95-9.

13. Saha A, Das NK, Hazra A, Gharami RC, Chowdhury SN, Datta PK. Cutaneous adverse drug reaction profile in a tertiary care outpatient setting in Eastern India. Indian J Pharmacol 2012; 44: 792-97.

14. Pudukadan D, Thappa DM. Adverse cutaneous drug reactions: Clinical pattern and causative agents in a tertiary care centre in South India. Indian J Dermatol Venereol Leprol 2004; 70: 20-24. 


\section{How to cite this article:}

Virendra Kumar, Vinod Jain, Dilip Kacchawaha, Pankaj Rao and Chouhan, C.P. 2020. Study of Clinico-Epidemiological Correlation and Clinical Pattern of Cutaneous Drug Reaction in Tertiary Level Hospital in Western Rajasthan. Int.J.Curr.Microbiol.App.Sci. 9(06): 2733-2742. doi: https://doi.org/10.20546/ijcmas.2020.906.332 